\title{
Dietary practices in nutritional transition: the case of Malaysian urban Chinese
}

\begin{abstract}
In this study involving 336 urban Chinese adults, more than $40 \%$ belong to at least the second generation in Malaysia. The result shows the persistence of several Chinese traditional dietary habits. Almost all the subjects take rice daily and an assortment of noodles weekly. A wide variety of vegetables are consumed frequently. Soup is consumed daily or weekly. Steaming and stir frying are preferred methods of cooking. Majority reported adding salt lightly in cooking, eating little fat on meat, and not adding sugar to tea or coffee, but they use sweetened condensed milk. Urban Malaysian Chinese do not appear to manifest a high-fat high-meat diet that is becoming characteristic of rapidly developing countries. Their dietary practices seem to reflect an inclination towards the "behavioural change" dietary pattern away from the "degenerative disease" pattern according to the concept of nutritional transition as described by Popkin.
\end{abstract}

Keyword: Dietary practices; Nutritional transition; Malaysian urban Chinese adults 